\title{
Weak Type Inequalities for Some Integral Operators on Generalized Nonhomogeneous Morrey Spaces
}

\author{
Hendra Gunawan, ${ }^{1}$ Denny Ivanal Hakim, ${ }^{1}$ Yoshihiro Sawano, ${ }^{2}$ and Idha Sihwaningrum ${ }^{3}$ \\ ${ }^{1}$ Department of Mathematics, Bandung Institute of Technology, Bandung 41032, Indonesia \\ ${ }^{2}$ Department of Mathematics and Information Sciences, Tokyo Metropolitan University, Minami-Ohsawa 1-1, \\ Hachioji, Tokyo 192-0397, Japan \\ ${ }^{3}$ Department of Mathematics, Jenderal Soedirman University, Purwokerto 53122, Indonesia
}

Correspondence should be addressed to Denny Ivanal Hakim; dnny_hkm@yahoo.com

Received 9 September 2013; Accepted 20 November 2013

Academic Editor: Vagif Guliyev

Copyright (C) 2013 Hendra Gunawan et al. This is an open access article distributed under the Creative Commons Attribution License, which permits unrestricted use, distribution, and reproduction in any medium, provided the original work is properly cited.

We prove weak type inequalities for some integral operators, especially generalized fractional integral operators, on generalized Morrey spaces of nonhomogeneous type. The inequality for generalized fractional integral operators is proved by using two different techniques: one uses the Chebyshev inequality and some inequalities involving the modified Hardy-Littlewood maximal operator and the other uses a Hedberg type inequality and weak type inequalities for the modified Hardy-Littlewood maximal operator. Our results generalize the weak type inequalities for fractional integral operators on generalized non-homogeneous Morrey spaces and extend to some singular integral operators. In addition, we also prove the boundedness of generalized fractional integral operators on generalized non-homogeneous Orlicz-Morrey spaces.

\section{Introduction}

In this paper, we prove that the theory of generalized Morrey spaces can be staged on the nondoubling setting on $\mathbb{R}^{d}$, so that we assume that $\mu$ is a positive Borel measure on $\mathbb{R}^{d}$ satisfying the growth condition; that is, there exist $n \in[0, d]$ and $C_{\mu}>0$ such that

$$
\mu(B(a, r)) \leq C_{\mu} r^{n}
$$

for any ball $B(a, r)$ centered at $a \in \mathbb{R}^{d}$ with radius $r>0$ (see [1-4]). For $0<n \leq d$ and a measurable function $\rho:(0, \infty) \rightarrow(0, \infty)$, we define the generalized fractional integral operator $I_{\rho}$ by

$$
I_{\rho} f(x)=\int_{\mathbb{R}^{d}} \frac{\rho(|x-y|)}{|x-y|^{n}} f(y) d \mu(y)
$$

for any suitable function $f$ on $\mathbb{R}^{d}$. This operator dates back to the book when $\rho(t)=t^{\alpha}$ for $0<\alpha<n$ [5, Section 6.1]. Note that if $\mu$ is the Lebesgue measure, then $I_{\rho}=I_{\alpha}$ is the fractional integral operator introduced in $[6,7]$. See also $[8$, 9] for exhaustive and comprehensive explanation about the operator. Below, we will always assume the Dini condition, that is, $\int_{0}^{1}(\rho(t) / t) d t<\infty$ and we also assume that $\rho$ satisfies the so-called growth condition; namely, there exist constants $C>0$ and $0<2 k_{1}<k_{2}<\infty$ such that

$$
\sup _{(r / 2)<s \leq r} \rho(s) \leq C \int_{k_{1} r}^{k_{2} r} \frac{\rho(t)}{t} d t
$$

for every $r>0$. For convenience, write $\rho^{*}(r)=\int_{k_{1} r}^{k_{2} r}(\rho(t) / t) d t$. Note that if $\rho$ satisfies the doubling condition, that is, there exists a constant $C>0$ such that $1 / C \leq \rho(r) / \rho(s) \leq$ $C$ whenever $1 / 2 \leq r / s \leq 2$, then $\rho$ satisfies the growth 
condition. See [10-13] for discussion about $I_{\rho}$, where $\rho$ satisfies the doubling condition.

Now, we say that a function $f$ belongs to the generalized nonhomogeneous Morrey space $L^{p, \phi}(\mu)=L^{p, \phi}\left(\mathbb{R}^{d}, \mu\right)$ for a function $\phi:(0, \infty) \rightarrow(0, \infty)$ and $1 \leq p<\infty$ if

$$
\begin{aligned}
& \|f\|_{L^{p, \phi}(\mu)} \\
& \quad:=\sup _{B(a, r) \subseteq \mathbb{R}^{d}} \frac{1}{\phi(r)}\left(\frac{1}{r^{n}} \int_{B(a, r)}|f(y)|^{p} d \mu(y)\right)^{1 / p}<\infty .
\end{aligned}
$$

Note that this definition is a special case of [14, Definition 1.1], where different types of operators are considered. In this paper, we will assume the following two conditions.

(1.a) The function $\phi$ is almost decreasing; that is, there exists a constant $C_{1}>0$ such that $\phi(r) \geq C_{1} \phi(s)$ for every $r \leq s$.

(1.b) The function $r \mapsto r^{n} \phi(r)^{p}$ is almost increasing; that is, there exists a constant $C_{2}>0$ such that $r^{n} \phi(r)^{p} \leq$ $C_{2} s^{n} \phi(s)^{p}$ for every $r \leq s$.

These two conditions imply that $\phi$ satisfies the doubling condition. Note that if $\phi(t)=t^{-n / p}$, then $L^{p, \phi}(\mu)=L^{p}(\mu)$ is the nonhomogeneous Lebesgue space.

The study of the boundedness of the fractional integral operator $I_{\alpha}$ on generalized Morrey spaces was initiated in [15, Theorem 3]. The following theorem presents the weak type inequalities for $I_{\alpha}$ on generalized nonhomogeneous Morrey spaces.

Theorem 1 (see [16, Theorem 2.4]). Let $1 \leq p<q<\infty$. Suppose that $\inf _{r>0} \phi(r)=0, \sup _{r>0} \phi(r)=\infty$, and there exist positive constants $C^{\prime}$ and $C^{\prime \prime}$ such that

$$
\begin{gathered}
\int_{r}^{\infty} \frac{\phi(t)^{p}}{t} d t \leq C^{\prime} \phi(r)^{p}, \\
r^{\alpha} \phi(r)+\int_{r}^{\infty} t^{\alpha-1} \phi(t) d t \leq C^{\prime \prime} \phi(r)^{p / q}
\end{gathered}
$$

for every $r>0$. Then, there exists a constant $C>0$ such that, for any function $f \in L^{p, \phi}(\mu)$ and any ball $B(a, r) \subseteq \mathbb{R}^{d}$, one has

$$
\mu\left(\left\{x \in B(a, r):\left|I_{\alpha} f(x)\right|>\gamma\right\}\right) \leq C r^{n} \phi(r)^{p}\left(\frac{\|f\|_{L^{p, \phi}(\mu)}}{\gamma}\right)^{q},
$$

for every $\gamma>0$.

Remark 2. Note that we can obtain the weak type inequalities for $I_{\alpha}$ on nonhomogeneous Lebesgue spaces which are proved in $[17,18]$ by taking $\phi(r)=r^{-n / p}$ and $1 / q=(1 / p)-$ $(\alpha / n)$ in Theorem 1 . By substituting $1 / q=1-(\alpha /(n-\lambda))$ for some $\lambda \in[0, n-\alpha)$ to $r^{\alpha} \phi(r) \leq C \phi(r)^{1 / q}$, we have $\int_{r}^{\infty} t^{\alpha-1} \phi(t) d t \leq C r^{\lambda+\alpha-n}$ for every $r>0$, which is one of the hypotheses in the weak type inequalities for $I_{\alpha}$ in [19].
The proof of Theorem 1 employs some inequalities involving the modified Hardy-Littlewood maximal operator $M^{n}$ (see [8]), which is defined for any locally integrable function $f$ by

$$
M^{n} f(x)=\sup _{r>0} \frac{1}{r^{n}} \int_{B(x, r)}|f(y)| d \mu(y)
$$

and the Chebyshev inequality which is presented in the following theorem.

Theorem 3 (see [20]). Let $E$ be a measurable subset of $\mathbb{R}^{d}$. If $f$ is an integrable function on $E$, then, for every $\gamma>0$, one has

$$
\mu(\{x \in E:|f(x)|>\gamma\}) \leq \frac{1}{\gamma} \int_{E}|f(x)| d \mu(x) .
$$

One of the reasons why we are fascinated with the generalized fractional integral operators is that these operators appear naturally in the context of differential equations; see [21, Section 6.4] for a nice explanation in connection with the holomorphic calculus of operators and see [22, (4.3)] and [23, Lemma 2.5] for a detailed account that $(1-\Delta)^{-\alpha / 2}$ with $\alpha>0$ satisfies the requirement of $\rho$ in the present paper. In addition, investigating generalized Morrey spaces is not a mere quest to the abstract theory; it arises naturally in the context of Sobolev embedding. In [24], the following proposition is proved.

Proposition 4 (see [24, Theorem 5.1]). Let $1<p<\infty$ and $0<\lambda<N$. Then, there exists a positive constant $C_{p, \lambda}$ such that

$$
\begin{aligned}
& \int_{B}|f(x)| d x \\
& \quad \leq C_{p, \lambda}|B|(1+|B|)^{-1 / p} \log \left(e+\frac{1}{|B|}\right)\left\|(1-\Delta)^{\lambda / 2 p} f\right\|_{L^{p, \lambda}}
\end{aligned}
$$

holds for all $f \in L^{p, \lambda}\left(\mathbb{R}^{N}\right)$ with $(1-\Delta)^{\lambda / 2 p} f \in L^{p, \lambda}\left(\mathbb{R}^{N}\right)$ and for all balls $B$, where $L^{p, \lambda}$ is the abbreviation of $L^{p, \varphi}$ with $\varphi(t)=$ $t^{-\lambda}$.

Later Proposition 4 is strengthened by [25, Example 5]. An example in [24] as well as the necessary and sufficient condition obtained in [25, Theorem 1.3] implicitly shows that the log factor above is absolutely necessary.

In this paper, we will prove the weak type inequalities for $I_{\rho}$ which is a generalization of Theorem 1 . In Section 2, we will prove the weak type inequalities for $I_{\rho}$ by using the Chebyshev inequality and some inequalities involving operator $M^{n}$. In Section 3, we will prove a Hedberg type inequality on generalized nonhomogeneous Morrey space by adapting the proof of a Hedberg type inequality on homogeneous setting in [25]. Through the weak type inequalities for $M^{n}$, we then prove the weak type inequalities for $I_{\rho}$ on generalized nonhomogeneous Morrey spaces. In Section 4, we extend our results to the singular integral operators defined in [1]. Finally, in Section 5, we prove the boundedness of $I_{\rho}$ 
on generalized nonhomogeneous Orlicz-Morrey spaces. See [26-28] for related results.

Throughout the paper, $C$ denotes a positive constant which is independent of the function $f$ and the variable $x$ and may have different values from line to line. We also denote by $C_{k}(k \in \mathbb{N})$ the fixed constants that satisfy certain conditions.

\section{Weak Type Inequalities for $I_{\rho}$ via the Chebyshev Inequality}

Now, we give an inequality which is used in the proof of the weak type inequalities for $I_{\rho}$ in the following lemma.

Lemma 5. Let $1 \leq p<q<\infty$. If $\rho$ and $\phi$ satisfy

$$
\int_{0}^{r} \frac{\rho(t)}{t} d t \leq C \phi(r)^{(p / q)-1}
$$

for every $r>0$, then, for any ball $B(x, R) \subseteq \mathbb{R}^{d}$ and every locally integrable function $f$, one has

$$
\int_{B(x, R)} \frac{\rho(|x-y|)}{|x-y|^{n}}|f(y)| d \mu(y) \leq C M^{n} f(x) \phi(R)^{(p / q)-1}
$$

Proof. Let $I(X)=\int_{(B, r)}\left(\rho(|x-y|) /|x-y|^{n}\right)|f(y)| d \mu(y)$, for any ball $B(x, R) \subseteq \mathbb{R}^{d}$. By the dyadic decomposition of the ball $B(x, R)$ and the growth condition of $\rho$, we have

$$
\begin{aligned}
I(x) & =\sum_{j=-\infty}^{-1} \int_{B\left(x, 2^{j+1} R\right) \backslash B\left(x, 2^{j} R\right)} \frac{\rho(|x-y|)}{|x-y|^{n}}|f(y)| d \mu(y) \\
& \leq C \sum_{j=-\infty}^{-1} \frac{\rho^{*}\left(2^{j+1} R\right)}{\left(2^{j} R\right)^{n}} \int_{B\left(x, 2^{j+1} R\right)}|f(y)| d \mu(y) \\
& \leq C M^{n} f(x) \sum_{j=-\infty}^{-1} \int_{k_{1} 2^{j+1} R}^{k_{2} 2^{j+1} R} \frac{\rho(t)}{t} d t .
\end{aligned}
$$

Then, we use the overlapping property (see $[29,30])$ to obtain

$$
\begin{aligned}
& \sum_{j=-\infty}^{-1} \int_{k_{1} 2^{j+1} R}^{k_{2} 2^{j+1} R} \frac{\rho(t)}{t} d t \\
& \quad=\sum_{j=-\infty}^{-1} \int_{0}^{k_{2} R} \chi_{\left[k_{1} 2^{j+1} R, k_{2} 2^{j+1} R\right]}(t) \frac{\rho(t)}{t} d t \\
& \quad=\int_{0}^{k_{2} R}\left(\sum_{j=-\infty}^{-1} \chi_{\left[k_{1} 2^{j+1} R, k_{2} 2^{j+1} R\right]}(t)\right) \frac{\rho(t)}{t} d t \\
& \quad \leq\left(1+\log _{2}\left(\frac{k_{2}}{k_{1}}\right)\right) \int_{0}^{k_{2} R} \frac{\rho(t)}{t} d t .
\end{aligned}
$$

If we use (10) and the doubling condition of $\phi$, then we have

$$
\sum_{j=-\infty}^{-1} \int_{k_{1} 2^{j+1} R}^{k_{2} 2^{j+1} R} \frac{\rho(t)}{t} d t \leq C \phi\left(k_{2} R\right)^{(p / q)-1} \leq C \phi(R)^{(p / q)-1} .
$$

Hence, $I(x) \leq C M^{n} f(x) \phi(R)^{(p / q)-1}$.

By letting $f \equiv 1$ or $f \equiv \chi_{B(a, r)}$, we have the following.

Corollary 6. Let $1 \leq p<q<\infty$ and $B(x, R)$ be any ball in $\mathbb{R}^{d}$. If the functions $\rho$ and $\phi$ satisfy inequality (10), then

$$
\int_{B(x, R)} \frac{\rho(|x-y|)}{|x-y|^{n}} d \mu(y) \leq C \phi(R)^{(p / q)-1}
$$

and, for any ball $B(a, r) \subseteq \mathbb{R}^{d}$, one has

$$
\begin{gathered}
\int_{B(x, R)} \frac{\rho(|x-y|)}{|x-y|^{n}} \chi_{B(a, r)}(y) d \mu(y) \\
\leq C M^{n} \chi_{B(a, r)}(x) \phi(R)^{(p / q)-1} .
\end{gathered}
$$

Remark 7. These two inequalities will be used later to prove one of our main theorems. The next lemma presents an inequality involving the modified Hardy-Littlewood maximal operator $M^{n}$. This inequality is an important part of the proof of the weak type inequalities for $I_{\alpha}$ in $[16,19]$. See [8] for similar results.

Lemma 8 (see [16]). Let $1 \leq p<\infty$. If $\phi$ satisfies $\int_{r}^{\infty}\left(\phi(t)^{p} / t\right) d t \leq C \phi(r)^{p}$ for everyr $>0$, then, for any function $f \in L^{p, \phi}(\mu)$ and any ball $B(a, r) \subseteq \mathbb{R}^{d}$, one has

$$
\int_{\mathbb{R}^{d}}|f(y)|^{p} M^{n} \chi_{B(a, r)}(y) d \mu(y) \leq C r^{n} \phi(r)^{p}\|f\|_{L^{p, \phi}(\mu)}^{p} .
$$

With Theorem 3, Corollary 6, and Lemma 8, we are now ready to prove the weak type inequalities for $I_{\rho}$ on generalized nonhomogeneous Morrey spaces.

Theorem 9 (see [31]). Let $1 \leq p<q<\infty$ and assume that $\sup _{r>0} \phi(r)=\infty$. If $\rho$ and $\phi$ satisfy

$$
\begin{gathered}
\int_{r}^{\infty} \frac{\phi(t)^{p}}{t} d t \leq C \phi(r)^{p}, \\
\phi(r) \int_{0}^{r} \frac{\rho(t)}{t} d t+\int_{r}^{\infty} \frac{\rho(t) \phi(t)}{t} d t \leq C \phi(r)^{p / q}
\end{gathered}
$$

for every $r>0$, then, for any function $f \in L^{p, \phi}(\mu)$ and any ball $B(a, r) \subseteq \mathbb{R}^{d}$, one has

$$
\mu\left(\left\{x \in B(a, r):\left|I_{\rho} f(x)\right|>\gamma\right\}\right) \leq C r^{n} \phi(r)^{p}\left(\frac{\|f\|_{L^{p, \phi}(\mu)}}{\gamma}\right)^{q},
$$

for every $\gamma>0$. 
Proof. Let $B(a, r)$ be any ball in $\mathbb{R}^{d}$. For every $x \in B(a, r)$ and $R>0$, let

$$
\begin{gathered}
I_{1}(x)=\int_{B(x, R)} \frac{\rho(|x-y|)}{|x-y|^{n}} f(y) d \mu(y), \\
I_{2}(x)=\int_{\mathbb{R}^{d} \backslash B(x, R)} \frac{\rho(|x-y|)}{|x-y|^{n}} f(y) d \mu(y) .
\end{gathered}
$$

Let $E_{\gamma}=\left\{x \in B(a, r):\left|I_{\rho} f(x)\right|>\gamma\right\}$, for any $\gamma>0$. Since $\left|I_{\rho} f(x)\right| \leq\left|I_{1}(x)\right|+\left|I_{2}(x)\right|$, we have

$$
\begin{aligned}
\mu\left(E_{\gamma}\right) \leq & \mu\left(\left\{x \in B(a, r):\left|I_{1}(x)\right|>\frac{\gamma}{2}\right\}\right) \\
& +\mu\left(\left\{x \in B(a, r):\left|I_{2}(x)\right|>\frac{\gamma}{2}\right\}\right) .
\end{aligned}
$$

By the dyadic decomposition of $\mathbb{R}^{d} \backslash B(x, R)$ and the growth condition of $\rho$, we have

$$
\begin{aligned}
\left|I_{2}(x)\right| & \leq \sum_{j=0}^{\infty} \int_{B\left(x, 2^{j+1} R\right) \backslash B\left(x, 2^{j} R\right)} \frac{\rho(|x-y|)}{|x-y|^{n}}|f(y)| d \mu(y) \\
& \leq C \sum_{j=0}^{\infty} \frac{\rho^{*}\left(2^{j+1} R\right)}{\left(2^{j} R\right)^{n}} \int_{B\left(x, 2^{j+1} R\right)}|f(y)| d \mu(y) .
\end{aligned}
$$

We use Hölder's inequality, the growth condition of $\mu$, and the definition of $\|f\|_{L^{p, \phi}(\mu)}$ to obtain

$$
\begin{aligned}
\left|I_{2}(x)\right| \leq C \sum_{j=0}^{\infty} \frac{\rho^{*}\left(2^{j+1} R\right)}{\left(2^{j} R\right)^{n}}\left(\int_{B\left(x, 2^{j+1} R\right)}|f(y)|^{p} d \mu(y)\right)^{1 / p} \\
\quad \times\left(\mu\left(B\left(x, 2^{j+1} R\right)\right)\right)^{1-(1 / p)} \\
\leq C \sum_{j=0}^{\infty} \rho^{*}\left(2^{j+1} R\right) \\
\quad \times\left(\frac{1}{\left(2^{j+1} R\right)^{n}} \int_{B\left(x, 2^{j+1} R\right)}|f(y)|^{p} d \mu(y)\right)^{1 / p} \\
\leq C\|f\|_{L^{p, \phi}(\mu)} \sum_{j=0}^{\infty} \rho^{*}\left(2^{j+1} R\right) \phi\left(2^{j+1} R\right) .
\end{aligned}
$$

By using the doubling condition of $\phi$ and the overlapping property, we have

$$
\begin{aligned}
& \sum_{j=0}^{\infty} \rho^{*}\left(2^{j+1} R\right) \phi\left(2^{j+1} R\right) \\
& \quad=\sum_{j=0}^{\infty} \phi\left(2^{j+1} R\right) \int_{2 k_{1} R}^{\infty} \chi_{\left[k_{1} 2^{j+1} R, k_{2} 2^{j+1} R\right]}(t) \frac{\rho(t)}{t} d t \\
& \quad \leq C \sum_{j=0}^{\infty} \int_{2 k_{1} R}^{\infty} \chi_{\left[k_{1} 2^{j+1} R, k_{2} 2^{j+1} R\right]}(t) \frac{\rho(t) \phi(t)}{t} d t \\
& =C \int_{2 k_{1} R}^{\infty}\left(\sum_{j=0}^{\infty} \chi_{\left[k_{1} 2^{j+1} R, k_{2} 2^{j+1} R\right]}(t)\right) \frac{\rho(t) \phi(t)}{t} d t \\
& \quad \leq C\left(1+\log _{2}\left(\frac{k_{2}}{k_{1}}\right)\right) \int_{2 k_{1} R}^{\infty} \frac{\rho(t) \phi(t)}{t} d t .
\end{aligned}
$$

Now, we invoke the integral assumption on $\rho \cdot \phi$ :

$$
\sum_{j=0}^{\infty} \rho^{*}\left(2^{j+1} R\right) \phi\left(2^{j+1} R\right) \leq C \phi\left(2 k_{1} R\right)^{p / q} \leq C \phi(R)^{p / q} .
$$

Hence,

$$
\left|I_{2}(x)\right| \leq C_{3}\|f\|_{L^{p, \phi}(\mu)} \phi(R)^{p / q} .
$$

Let $\tilde{\gamma}=\left(\gamma / 2 C_{3}\|f\|_{L^{p, \phi}(\mu)}\right)^{q / p}$. Remark that $\int_{1}^{\infty}\left(\phi(t)^{p} / t\right) d t \leq$ $C \phi(1)^{p}$ implies that inf $r>0 \phi(r)=0$. Otherwise,

$$
\infty=\inf _{r^{\prime}>0} \phi\left(r^{\prime}\right)^{p} \int_{1}^{\infty} \frac{1}{t} d t \leq \int_{1}^{\infty} \frac{\phi(t)^{p}}{t} d t \leq C \phi(1)^{p},
$$

which is impossible. Now, from $\inf _{r>0} \phi(r)=0<\tilde{\gamma}<\infty=$ $\sup _{r>0} \phi(r)$, we can find $k_{0} \in \mathbb{Z}$ such that, for $R_{1}=2^{k_{0}}$ and $R_{2}=2^{k_{0}-1}$, we have

$$
\phi\left(R_{1}\right) \leq \tilde{\gamma} \leq \phi\left(R_{2}\right) .
$$

Since $R_{1} / R_{2}=2$, there exists $C>0$ such that $\phi\left(R_{2}\right) \leq C \phi\left(R_{1}\right)$. Hence,

$$
\phi\left(R_{1}\right) \leq \tilde{\gamma} \leq C \phi\left(R_{1}\right) .
$$

Taking $R=R_{1}$, we obtain

$$
\left|I_{2}(x)\right| \leq C_{3}\|f\|_{L^{p, \phi}(\mu)} \phi\left(R_{1}\right)^{p / q} \leq C_{3}\|f\|_{L^{p, \phi}(\mu)} \tilde{\gamma}^{p / q} \leq \frac{\gamma}{2} .
$$

Consequently,

$$
\mu\left(E_{\gamma}\right) \leq \mu\left(\left\{x \in B(a, r):\left|I_{1}(x)\right|>\frac{\gamma}{2}\right\}\right) .
$$


We combine Hölder's inequality and the inequality (15) to obtain

$$
\begin{aligned}
\left|I_{1}(x)\right| \leq & \left(\int_{B\left(x, R_{1}\right)} \frac{\rho(|x-y|)}{|x-y|^{n}}|f(y)|^{p} d \mu(y)\right)^{1 / p} \\
& \times\left(\int_{B\left(x, R_{1}\right)} \frac{\rho(|x-y|)}{|x-y|^{n}} d \mu(y)\right)^{1-(1 / p)} \\
\leq & C_{4} \phi\left(R_{1}\right)^{((p / q)-1)(1-(1 / p))} \\
& \times\left(\int_{B\left(x, R_{1}\right)} \frac{\rho(|x-y|)}{|x-y|^{n}}|f(y)|^{p} d \mu(y)\right)^{1 / p} .
\end{aligned}
$$

Finally, by using the last inequality and the Chebyshev inequality, we get

$$
\begin{aligned}
& \mu\left(E_{\gamma}\right) \leq \mu\left\{x \in B(a, r): \int_{B\left(x, R_{1}\right)} \frac{\rho(|x-y|)}{|x-y|^{n}}|f(y)|^{p} d \mu(y)\right. \\
&>\left.\frac{(\gamma / 2)^{p}}{C_{4}^{p} \phi\left(R_{1}\right)^{((p / q)-1)(p-1)}}\right\} \\
& \leq \frac{2^{p} C_{4}^{p} \phi\left(R_{1}\right)^{((p / q)-1)(p-1)}}{\gamma^{p}} \\
& \times \int_{B(a, r)} \int_{B\left(x, R_{1}\right)} \frac{\rho(|x-y|)}{|x-y|^{n}|f(y)|^{p} d \mu(y) d \mu(x)} \\
&= \frac{C}{\gamma^{p}} \phi\left(R_{1}\right)^{((p / q)-1)(p-1)} \\
& \times \int_{\mathbb{R}^{d}} \int_{B\left(x, R_{1}\right)} \frac{\rho(|x-y|)|f(y)|^{p}}{|x-y|^{n}} \chi_{B(a, r)}(\chi) \\
& \times(x) d \mu(y) d \mu(x) .
\end{aligned}
$$

By virtue of the inequalities (16), (17), and (29) as well as the definition of $\tilde{\gamma}$, we get

$$
\begin{aligned}
\mu\left(E_{\gamma}\right) \leq & \frac{C}{\gamma^{p}} \phi\left(R_{1}\right)^{((p / q)-1)(p-1)} \int_{\mathbb{R}^{d}}|f(y)|^{p} \\
& \times \int_{B\left(y, R_{1}\right)} \frac{\rho(|x-y|)}{|x-y|^{n}} \chi_{B(a, r)}(x) d \mu(x) d \mu(y) \\
\leq & \frac{C}{\gamma^{p}} \phi\left(R_{1}\right)^{((p / q)-1) p} \int_{\mathbb{R}^{d}}|f(y)|^{p} M^{n} \chi_{B(a, r)}(y) d \mu(y) \\
\leq & \frac{C}{\gamma^{p}} \tilde{\gamma}^{\left(p^{2} / q\right)-p} r^{n} \phi(r)^{p}\|f\|_{L^{p, \phi}(\mu)}^{p} \\
\leq & C r^{n} \phi(r)^{p}\left(\frac{\|f\|_{L^{p, \phi}(\mu)}}{\gamma}\right)^{q},
\end{aligned}
$$

as desired.
Remark 10. Note that $\rho(t)=t^{\alpha}$, where $0<\alpha<n$ satisfies the condition of Theorem 9 and, for this $\rho$, we obtain the weak type inequalities for $I_{\alpha}$ in Theorem 1 .

\section{Weak Type Inequalities for $I_{\rho}$ via a Hedberg Type Inequality and Weak Type Inequalities for $M^{n}$}

In this section, we will prove weak type inequalities for $I_{\rho}$ using a different technique, namely, via a Hedberg type inequality and weak type inequalities for $M^{n}$. It turns out that some hypotheses can be removed. The Hedberg type inequality is presented in the following proposition.

Proposition 11 (see $[25,31])$. Let $1 \leq p<q<\infty$. If $\rho$ and $\phi$ satisfy

$$
\phi(r) \int_{0}^{r} \frac{\rho(t)}{t} d t+\int_{r}^{\infty} \frac{\rho(t) \phi(t)}{t} d t \leq C \phi(r)^{p / q}
$$

for every $r>0$, then, for every $f \in L^{p, \phi}(\mu)$ and $x \in \mathbb{R}^{d}$, one has

$$
\left|I_{\rho} f(x)\right| \leq C\left(M^{n} f(x)^{p / q}\|f\|_{L^{p, \phi}(\mu)}^{1-(p / q)}+\|f\|_{L^{p, \phi}(\mu)} \inf _{r>0} \phi(r)^{p / q}\right) .
$$

Proof. We adapt the proof of a Hedberg type inequality on generalized Morrey space in [25]. For every $x \in \mathbb{R}^{d}$ and $R>$ 0 , write $I_{\rho} f(x)=I_{1}(x)+I_{2}(x)$, where $I_{1}(x)$ and $I_{2}(x)$ are defined in the proof of Theorem 9. By using the inequalities (11) and (26), we get

$$
\left|I_{\rho} f(x)\right| \leq C\left(M^{n} f(x) \phi(R)^{(p / q)-1}+\|f\|_{L^{p, \phi}(\mu)} \phi(R)^{p / q}\right) .
$$

Next, we separate the proof into the following two cases.

First Case $\left(M^{n} f(x) \leq 2 C_{\mu}^{1-(1 / p)}\|f\|_{L^{p, \phi}(\mu)} \inf _{r>0} \phi(r)\right)$. In this case, we have

$$
\begin{array}{rl}
\left|I_{\rho} f(x)\right| \leq C & \left(\left(2 C_{\mu}^{1-(1 / p)}\|f\|_{L^{p, \phi}(\mu)} \phi(R)\right) \phi(R)^{(p / q)-1}\right. \\
& \left.+\|f\|_{L^{p, \phi}(\mu)} \phi(R)^{p / q}\right) \\
\leq C & f \|_{L^{p, \phi}(\mu)} \phi(R)^{p / q}
\end{array}
$$

for every $R>0$. Hence, $\left|I_{\rho} f(x)\right| \leq C\|f\|_{L^{p, \phi}(\mu)} \inf _{r>0} \phi(r)^{p / q}$.

Second Case. $M^{n} f(x)>2 C_{\mu}^{1-(1 / p)}\|f\|_{L^{p, \phi}(\mu)} \inf _{r>0} \phi(r)$. We use Hölder's inequality, the growth condition of $\mu$, and the definition of $\|f\|_{L^{p, \phi}(\mu)}$ to obtain

$$
\begin{aligned}
& \frac{1}{R^{n}} \int_{B(x, R)}|f(y)| d \mu(y) \\
& \quad \leq \frac{1}{R^{n}}\left(\int_{B(x, R)}|f(y)|^{p} d \mu(y)\right)^{1 / p} \mu(B(x, R))^{1-(1 / p)} \\
& \quad \leq C_{\mu}^{1-(1 / p)}\|f\|_{L^{p, \phi}(\mu)} \phi(R)
\end{aligned}
$$


for every $R>0$. Hence,

$$
M^{n} f(x) \leq C_{\mu}^{1-(1 / p)}\|f\|_{L^{p, \phi}(\mu)} \sup _{r>0} \phi(r) .
$$

Since $\sup _{r>0} \phi(r)>0$, we have

$$
\inf _{r>0} \phi(r)<\frac{M^{n} f(x)}{2 C_{\mu}^{1-(1 / p)}\|f\|_{L^{p, \phi}(\mu)}} \leq \frac{1}{2} \sup _{r>0} \phi(r)<\sup _{r>0} \phi(r) .
$$

Thus, there exists $j_{0} \in \mathbb{Z}$ such that

$$
\phi\left(R_{1}\right) \leq \frac{M^{n} f(x)}{2 C_{\mu}^{1-(1 / p)}\|f\|_{L^{p, \phi}(\mu)}} \leq \phi\left(R_{2}\right)
$$

for $R_{1}=2^{j_{0}}$ and $R_{2}=2^{j_{0}-1}$. Since $R_{1} / R_{2}=2$, there exists $C>0$ such that

$$
\phi\left(R_{1}\right) \leq \frac{M^{n} f(x)}{2 C_{\mu}^{1-(1 / p)}\|f\|_{L^{p, \phi}(\mu)}} \leq C \phi\left(R_{1}\right) .
$$

By choosing $R=R_{1}$ in the inequality (37) and using the inequality (43), we have

$$
\begin{aligned}
& \left|I_{\rho} f(x)\right| \\
& \leq C\left(\left(2 C_{\mu}^{1-(1 / p)}\|f\|_{L^{p, \phi}(\mu)} C \phi\left(R_{1}\right)\right) \phi\left(R_{1}\right)^{(p / q)-1}\right. \\
& \left.\quad+\|f\|_{L^{p, \phi}(\mu)} \phi\left(R_{1}\right)^{p / q}\right) \\
& \leq C \phi\left(R_{1}\right)^{p / q}\|f\|_{L^{p, \phi}(\mu)} \\
& \leq C\left(\frac{M^{n} f(x)}{2 C_{\mu}^{1-(1 / p)}\|f\|_{L^{p, \phi}(\mu)}}\right)^{p / q}\|f\|_{L^{p, \phi}(\mu)} \\
& \leq C M^{n} f(x)^{p / q}\|f\|_{L^{p, \phi}(\mu)}^{1-(p / q)} .
\end{aligned}
$$

From these two cases, we obtain the inequality (36).

Sihwaningrum et al. [19] proved the weak type inequalities for $M^{n}$ on generalized nonhomogeneous Morrey space by assuming that $\phi^{p}$ satisfies the integral condition; that is, $\int_{r}^{\infty}\left(\phi(t)^{p} / t\right) d t \leq C \phi(r)^{p}$ for every $r>0$. In [19], the weak type inequalities for $I_{\alpha}$ are also proved by using the weak type inequalities for $M^{n}$. In this paper, we remove the integral condition of $\phi^{p}$ in the hypothesis of our proposition below. See [32, Theorem 2.3] and [33, Theorem 2.3] for such attempts.

Proposition 12 (see [31]). Let $1 \leq p<\infty$; then, there exists a constant $C>0$ such that, for any function $f \in L^{p, \phi}(\mu)$ and any ball $B(a, r) \subseteq \mathbb{R}^{d}$, one has

$$
\mu\left\{x \in B(a, r): M^{n} f(x)>\gamma\right\} \leq C r^{n} \phi(r)^{p}\left(\frac{\|f\|_{L^{p, \phi}(\mu)}}{\gamma}\right)^{p},
$$

for every $\gamma>0$.
When $p>1$, we have the strong boundedness; see $[15$, Theorem 1] and [34, Lemma 2.4] for the Lebesgue case and see [35, Theorem 4.3] for the strong $L^{p, \phi_{1}}(\mu)$ to $L^{p, \phi_{2}}(\mu)$ result and the weak $L^{p, \phi_{1}}(\mu)$ to $L^{p, \phi_{2}}(\mu)$ result with $\mu$ equal to the Lebesgue measure.

Proof. The proof is similar to that of strong boundedness of maximal operator on generalized nonhomogeneous Morrey spaces which is discussed in [34]. The difference is that in the final step we use the Chebyshev inequality, as we will see below. Consider the ball $B(a, r) \subseteq \mathbb{R}^{d}$. Let $x \in B(a, r)$ and let $\gamma$ be any positive real number. For $y \in \mathbb{R}^{d}$, define $f_{1}(y)=\chi_{B(a, 2 r)}(y) f(y)$ and $f_{2}(y)=f(y)-f_{1}(y)$. Note that

$$
M^{n} f_{2}(x)=\sup _{R>0} \frac{1}{R^{n}} \int_{B(x, R)}\left(1-\chi_{B(a, 2 r)}(y)\right)|f(y)| d \mu(y) .
$$

Since $B(x, R) \subseteq B(a, 2 r)$, for every $R<r$, we have

$$
\frac{1}{R^{n}} \int_{B(x, R)}\left(1-\chi_{B(a, 2 r)}(y)\right)|f(y)| d \mu(y)=0
$$

for every $R<r$. Hence,

$$
M^{n} f_{2}(x) \leq \sup _{R>r} \frac{1}{R^{n}} \int_{B(x, R)}|f(y)| d \mu(y) .
$$

Observe that, for every $R>r$, we have

$$
\begin{aligned}
& \frac{1}{\phi(r) R^{n}} \int_{B(x, R)}|f(y)| d \mu(y) \\
& \quad \leq \frac{C}{\phi(R) R^{n}}\left(\int_{B(x, R)}|f(y)|^{p} d \mu(y)\right)^{1 / p}(\mu(B(x, R)))^{1-(1 / p)} \\
& \quad \leq \frac{C}{\phi(R)}\left(\frac{1}{R^{n}} \int_{B(x, R)}|f(y)|^{p} d \mu(y)\right)^{1 / p} \\
& \quad \leq C\|f\|_{L^{p, \phi}(\mu)} .
\end{aligned}
$$

Thus,

$$
M^{n} f_{2}(x) \leq C \phi(r)\|f\|_{L^{p, \phi}(\mu)} .
$$

Since $M^{n} f(x) \leq M^{n} f_{1}(x)+M^{n} f_{2}(x)$, we have

$$
\begin{aligned}
\mu\{x & \left.\in B(a, r): M^{n} f(x)>\gamma\right\} \\
\leq & \mu\left\{x \in B(a, r): M^{n} f_{1}(x)>\frac{\gamma}{2}\right\} \\
& +\mu\left\{x \in B(a, r): M^{n} f_{2}(x)>\frac{\gamma}{2}\right\} .
\end{aligned}
$$


For the first term, we use the weak type inequalities for $M^{n}$ on the nonhomogeneous Lebesgue space $L^{p}(\mu)$ (see [18]) to obtain

$$
\begin{gathered}
\mu\left\{x \in B(a, r): M^{n} f_{1}(x)>\frac{\gamma}{2}\right\} \\
\leq C\left(\frac{\left\|f_{1}\right\|_{L^{p}(\mu)}}{\gamma}\right)^{p} \\
\leq \frac{C}{\gamma^{p}} \int_{B(a, 2 r)}|f(y)|^{p} d \mu(y) \\
\leq \frac{C}{\gamma^{p}} \phi(2 r)^{p}(2 r)^{n}\|f\|_{L^{p, \phi}(\mu)}^{p} \\
\leq C r^{n} \phi(r)^{p}\left(\frac{\|f\|_{L^{p, \phi}(\mu)}}{\gamma}\right)^{p} .
\end{gathered}
$$

Meanwhile, for the second term, by using the Chebyshev inequality and the inequality (50), we have

$$
\begin{aligned}
\mu\{x & \left.\in B(a, r): M^{n} f_{2}(x)>\frac{\gamma}{2}\right\} \\
& =\mu\left\{x \in B(a, r): M^{n} f_{2}(x)^{p}>\left(\frac{\gamma}{2}\right)^{p}\right\} \\
& \leq \frac{2^{p}}{\gamma^{p}} \int_{B(a, r)} M^{n} f_{2}(x)^{p} d \mu(x) \\
& \leq \frac{2^{p}}{\gamma^{p}} \int_{B(a, r)} C^{p} \phi(r)^{p}\|f\|_{L^{p, \phi}(\mu)}^{p} d \mu(x) \\
& \leq \frac{2^{p}}{\gamma^{p}} C^{p} \phi(r)^{p}\|f\|_{L^{p, \phi}(\mu)}^{p}(B(a, r)) \\
& \leq C r^{n} \phi(r)^{p}\left(\frac{\|f\|_{L^{p, \phi}(\mu)}}{\gamma}\right)^{p} .
\end{aligned}
$$

Finally, by combining these two estimates, we obtain inequality (45).

With Propositions 11 and 12, we are now ready to prove the weak type inequalities for $I_{\rho}$ on generalized nonhomogeneous Morrey spaces.

Theorem 13 (see [31]). Let $1 \leq p<q<\infty$. If $\rho$ and $\phi$ satisfy inequality (35), then, for any function $f \in L^{p, \phi}(\mu)$ and any ball $B(a, r) \subseteq \mathbb{R}^{d}$, one has

$$
\mu\left(\left\{x \in B(a, r):\left|I_{\rho} f(x)\right|>\gamma\right\}\right) \leq C r^{n} \phi(r)^{p}\left(\frac{\|f\|_{L^{p, \phi}(\mu)}}{\gamma}\right)^{q}
$$

for every $\gamma>0$.
Proof. This proof is adapted from [25]. We replace $\gamma$ by $2 \gamma$. Consider the ball $B(a, r) \subseteq \mathbb{R}^{d}$. By applying Proposition 11, we have

$$
\begin{aligned}
\mu\{x \in & \left.B(a, r):\left|I_{\rho} f(x)\right|>2 \gamma\right\} \\
\leq \mu & \left\{x \in B(a, r): C\left(M^{n} f(x)^{p / q}\|f\|_{L^{p, \phi}(\mu)}^{1-(p / q)}\right.\right. \\
& \left.\left.+\|f\|_{L^{p, \phi}(\mu)} \inf _{r>0} \phi(r)^{p / q}\right)>2 \gamma\right\} \\
\leq \mu & \left\{x \in B(a, r): C M^{n} f(x)^{p / q}\|f\|_{L^{p, \phi}(\mu)}^{1-(p / q)}>\gamma\right\} \\
+ & \mu\left\{x \in B(a, r): C\|f\|_{L^{p, \phi}(\mu)} \inf _{r>0} \phi(r)^{p / q}>\gamma\right\} .
\end{aligned}
$$

Observe that the second term in the most right-hand side of the above inequality vanishes, when

$$
C\|f\|_{L^{p, \phi}(\mu)} \inf _{r>0} \phi(r)^{p / q} \leq \gamma
$$

So, to estimate the term, we can suppose that

$$
C\|f\|_{L^{p, \phi}(\mu)} \inf _{r>0} \phi(r)^{p / q}>\gamma
$$

With this in mind, we calculate

$$
\begin{gathered}
\mu\left\{x \in B(a, r): C\|f\|_{L^{p, \phi}(\mu)} \inf _{r>0} \phi(r)^{p / q}>\gamma\right\} \\
=\mu(B(a, r)) \leq C r^{n} \phi(r)^{p}\left(\frac{\|f\|_{L^{p, \phi}(\mu)}}{\gamma}\right)^{q} .
\end{gathered}
$$

Meanwhile, by using Proposition 12, we have

$$
\begin{aligned}
& \mu\left\{x \in B(a, r): C M^{n} f(x)^{p / q}\|f\|_{L^{p, \phi}(\mu)}^{1-(p / q)}>\gamma\right\} \\
& \leq \mu\left\{x \in B(a, r): M^{n} f(x)>\left(\frac{\gamma}{C\|f\|_{L^{p, \phi}(\mu)}^{1-(p / q)}}\right)^{q / p}\right\} \\
& \leq C r^{n} \phi(r)^{p}\|f\|_{L^{p, \phi}(\mu)}^{p}\left(\frac{\gamma}{C\|f\|_{L^{p, \phi}(\mu)}^{1-(p / q)}}\right)^{-q} \\
& \leq C r^{n} \phi(r)^{p}\left(\frac{\|f\|_{L^{p, \phi}(\mu)}}{\gamma}\right)^{q} .
\end{aligned}
$$

By summing the two previous estimates, we get the desired inequality.

Remarks 1. (i) Note that the hypotheses $\int_{r}^{\infty}\left(\phi(t)^{p} / t\right) d t \leq$ $C \phi(r)^{p}$ in Theorem 9 are not included in Theorem 13, since we can prove the weak type inequalities for $M^{n}$ without this condition. 
(ii) The conditions on $\phi$, namely, $\inf _{r>0} \phi(r)=0$ and $\sup _{r>0} \phi(r)=\infty$, are not included in the hypotheses in Theorem 13. However, we have to use the weak type inequalities for $M^{n}$ on generalized nonhomogeneous Morrey spaces and a Hedberg type inequality for $I_{\rho}$ in the proof of Theorem 13.

\section{Boundedness of Singular Integral Operators}

Proposition 12 carries over to the singular integral operator whose definition is given in [1]. Recall that the singular integral operator $T$ is a bounded linear operator on $L^{2}(\mu)$ for which there exists a function $K$ that satisfies three properties listed below.

(4.a) There exists $C>0$ such that $|K(x, y)| \leq C /|x-y|^{n}$ for all $x \neq y$.

(4.b) There exist $\varepsilon>0$ and $C>0$ such that

$$
|K(x, y)-K(z, y)|+|K(y, x)-K(y, z)| \leq C \frac{|x-z|^{\varepsilon}}{|x-y|^{n+\varepsilon}}
$$

$$
\text { if }|x-y| \geq 2|x-z| \text { with } x \neq y \text {. }
$$

(4.c) If $f$ is a bounded $\mu$-measurable function with a compact support, then we have

$$
T f(x)=\int_{\mathbb{R}^{d}} K(x, y) f(y) d \mu(y) \quad \forall x \notin \operatorname{supp}(f) .
$$

As for this singular integral operator $T$, the following result is due to Nazarov, Treil, and Volberg.

Proposition 14 (see $[1,2])$. The singular operator $T$ is bounded on $L^{p}(\mu)$ for $1<p<\infty$. Moreover, there exists a constant $C>0$ such that

$$
\mu\left\{x \in \mathbb{R}^{d}:|T f(x)|>\gamma\right\} \leq C \frac{\|f\|_{L^{1}(\mu)}}{\gamma}
$$

for every $f \in L^{1}(\mu)$ and every $\gamma>0$.

Theorem 15. Let $T$ be a singular integral operator. Let $1 \leq p<$ $\infty$. In addition to the doubling condition, assume that

$$
\int_{r}^{\infty} \frac{\phi(t)}{t} d t \leq C \phi(r)
$$

for every $r>0$. Then, there exists a constant $C>0$ such that, for any function $f \in L^{p, \phi}(\mu)$ and any ball $B(a, r) \subseteq \mathbb{R}^{d}$, one has

$$
\mu\{x \in B(a, r):|T f(x)|>\gamma\} \leq C r^{n} \phi(r)^{p}\left(\frac{\|f\|_{L^{p, \phi}(\mu)}}{\gamma}\right)^{p}
$$

for every $\gamma>0$.
Proof. The proof is a modification of that of Proposition 12. We decompose $f=f_{1}+f_{2}$ as before. The treatment of $f_{1}$ is the same as that in Proposition 12 but by using the weak type inequality for $T$ in Proposition 14. We need to take care of $f_{2}$. By the condition (4.a), Hölder's inequality, and the growth condition of $\mu$, we have

$$
\begin{aligned}
\left|T f_{2}(x)\right| & \leq C \int_{\mathbb{R}^{d} \backslash B(x, r)} \frac{|f(y)|}{|x-y|^{n}} d \mu(y) \\
& \leq C \sum_{j=0}^{\infty} \frac{1}{\left(2^{j} r\right)^{n}} \int_{B\left(x, 2^{j+1} r\right)}|f(y)| d \mu(y) \\
& \leq C \sum_{j=0}^{\infty} \frac{1}{\left(2^{j} r\right)^{n}}\left(\int_{B\left(x, 2^{j+1} r\right)}|f(y)|^{p} d \mu(y)\right)^{1 / p} \\
& \leq C \sum_{j=0}^{\infty}\left(\frac{1}{\left(2^{j+1} r\right)^{n}} \int_{B\left(x, 2^{j+1} r\right)}|f(y)|^{p} d \mu(y)\right)^{1 / p} \\
& \left.\left.\leq C \sum_{j=0}^{\infty} \phi\left(2^{j+1} r\right)\|f\|_{L^{p, \phi}(\mu)}\left(x, 2^{j+1} r\right)\right)\right)^{1-(1 / p)} \\
& \leq C \|_{L^{p, \phi}(\mu)} \int_{r}^{\infty} \frac{\phi(t)}{t} d t .
\end{aligned}
$$

If we use our integrability assumption, then we have a pointwise estimate:

$$
\left|T f_{2}(x)\right| \leq C \phi(r)\|f\|_{L^{p, \phi}(\mu)}
$$

So, we are done.

Remark 16. If we define the generalized weak Morrey space of nonhomogeneous type $w L^{p, \phi}(\mu)$ to be the set of all $\mu$ measurable functions $f$ such that

$\|f\|_{w L^{p, \phi}(\mu)}$

$$
:=\sup _{B(a, r) \subseteq \mathbb{R}^{d}, \gamma>0} \frac{\gamma(\mu\{x \in B(a, r):|f(x)|>\gamma\})^{1 / p}}{r^{n / p} \phi(r)}<\infty,
$$

then the inequality (64) amounts to the boundedness of $T$ from $L^{p, \phi}(\mu)$ to $w L^{p, \phi}(\mu)$. Similarly, our previous results can be translated into this language. In the following section, we will use these notations for convenience.

\section{Generalized Nonhomogeneous Orlicz-Morrey Spaces}

Our results above can be carried over to generalized nonhomogeneous Orlicz-Morrey spaces. We first formulate our main results and then prove them later in Sections 5.1-5.3. 
Recall that $\Phi:[0, \infty) \rightarrow[0, \infty)$ is a Young function, if $\Phi$ is bijective and convex. We define the $\Phi$-average of $f$ over a ball $B(a, r)$ as follows:

$$
\begin{aligned}
& \|f\|_{\Phi, B(a, r)} \\
& \quad=\inf \left\{\lambda>0: \frac{1}{r^{n}} \int_{B(a, r)} \Phi\left(\frac{|f(y)|}{\lambda}\right) d \mu(y) \leq 1\right\} .
\end{aligned}
$$

The generalized nonhomogeneous Orlicz-Morrey space $L^{\Phi, \phi}(\mu)=L^{\Phi, \phi}\left(\mathbb{R}^{d}, \mu\right)$ is the set of all $f \in L_{\text {loc }}^{1}(\mu)$ for which the norm

$$
\|f\|_{L^{\Phi, \phi}(\mu)}=\sup _{B(a, r) \subseteq \mathbb{R}^{d}} \frac{1}{\phi(r)}\|f\|_{\Phi, B(a, r)}
$$

is finite. Note that if $\Phi(t)=t^{p}$, then $L^{\Phi, \phi}(\mu)=L^{p, \phi}(\mu)$. About the structure of this function space, we have the following.

Theorem 17. Let $\Phi:[0, \infty) \rightarrow[0, \infty)$ be a Young function and let $\phi$ satisfy the two conditions (1.a) and (1.b) as usual. Then, $L^{\Phi, \phi}(\mu)$ is a Banach space.

We define the generalized weak Orlicz-Morrey spaces of nonhomogeneous type as follows. For a Young function $\Phi$, the generalized weak Orlicz-Morrey space of nonhomogeneous type $w L^{\Phi, \phi}(\mu)=w L^{\Phi, \phi}\left(\mathbb{R}^{d}, \mu\right)$ is the set of all $\mu$ measurable functions $f$ for which the norm

$$
\|f\|_{w L^{\Phi, \phi}(\mu)}=\sup _{B(a, r) \subseteq \mathbb{R}^{d}, \gamma>0} \frac{\gamma}{\phi(r)}\left\|\chi_{\{|f|>\gamma\}}\right\|_{\Phi, B(a, r)}
$$

is finite. Write $w L^{p, \phi}(\mu)=w L^{\Phi, \phi}(\mu)$, when $\Phi(t)=t^{p}$. It is not so hard to prove

$$
\|f\|_{w L^{\Phi, \phi}(\mu)} \leq\|f\|_{L^{\Phi, \phi}(\mu)}
$$

for all $\mu$-measurable functions $f$ from the inequality

$$
\gamma \chi_{\{|f|>\gamma\}} \leq|f| \text {. }
$$

By taking $f(x)=(1 /|x|) \chi_{[-1,1]}(x)$ and $\phi(t)=(1 / t) \log (3+$ $t)$ and $\mu$ is the Lebesgue measure on $\mathbb{R}$, we see that $f \in$ $w L^{1, \phi}(\mathbb{R}, \mu) \backslash L^{1, \phi}(\mathbb{R}, \mu)$, showing that $w L^{1, \phi}(\mathbb{R}, \mu)$ is a proper superset of $L^{1, \phi}(\mathbb{R}, \mu)$.

Theorem 18. Let $\Phi:[0, \infty) \rightarrow[0, \infty)$ be a Young function and let $\phi$ satisfy the two conditions (1.a) and (1.b) as usual. Then, $w L^{\Phi, \phi}(\mu)$ is a quasi-Banach space. More precisely,

(1) $\|f\|_{w L^{\Phi, \phi}(\mu)}=0$ if and only if $f=0$;

(2) $\|c f\|_{w L^{\Phi, \phi}(\mu)}=|c|\|f\|_{w L^{\Phi, \phi}(\mu)}$ for all $c \in \mathbb{C}$ and $f \in$ $w L^{\Phi, \phi}(\mu)$

(3) if $\left\{f_{k}\right\}_{k=1}^{\infty}$ is a sequence in $w L^{\Phi, \phi}(\mu)$ such that

$$
\lim _{k_{1}, k_{2} \rightarrow \infty}\left\|f_{k_{1}}-f_{k_{2}}\right\|_{w L^{\Phi, \phi}(\mu)}=0 .
$$

Then, there exists $g \in w L^{\Phi, \phi}(\mu)$ such that

$$
\lim _{k \rightarrow \infty}\left\|g-f_{k}\right\|_{w L^{\Phi, \phi}(\mu)}=0 .
$$

We prove the following boundedness result on generalized nonhomogeneous Orlicz-Morrey spaces.

Theorem 19. Let $\Phi:[0, \infty) \rightarrow[0, \infty)$ be a Young function and let $\phi$ satisfy the two conditions (1.a) and (1.b) as usual. Then, the maximal operator $M$ is bounded from $L^{\Phi, \phi}(\mu)$ to $w L^{\Phi, \phi}(\mu)$. If we assume that

$$
\int_{R}^{\infty} \frac{\phi(t)}{t} d t \leq C \phi(R) \quad(R>0)
$$

and that $\Phi$ satisfies the doubling condition, then the singular integral operator $T$ is bounded from $L^{\Phi, \phi}(\mu)$ to $w L^{\Phi, \phi}(\mu)$.

Theorem 20. Let $\rho:(0, \infty) \rightarrow(0, \infty)$ and, for some $b \in$ $(0,1], \phi:(0, \infty) \rightarrow(0, \infty)$, satisfy

$$
\phi(r) \int_{0}^{r} \frac{\rho(t)}{t} d t+\int_{0}^{r} \frac{\phi(t) \rho(t)}{t} d t \leq C \phi(r)^{b}
$$

for every $r>0$. Suppose that $\Phi:[0, \infty) \rightarrow[0, \infty)$ is a Young function with the doubling condition. Set

$$
\psi(t)=\phi(t)^{b}, \quad \Psi(t)=\Phi\left(t^{1 / b}\right), \quad t \in[0, \infty) .
$$

Then,

$$
\left\|I_{\rho} f\right\|_{w L^{\Psi, \psi}(\mu)} \leq C\|f\|_{L^{\Phi, \phi}(\mu)} .
$$

5.1. Proof of Theorems 17 and 18. We start with a lemma.

Lemma 21. Let $\Phi:[0, \infty) \rightarrow[0, \infty)$ be a Young function with the doubling property:

$$
\Phi(2 t) \leq C \Phi(t), \quad t \in[0, \infty)
$$

For $\mu$-measurable functions $f$ and $g$ and a ball $B(a, r)$, one has

$$
\|f+g\|_{\Phi, B(a, r)} \leq\|f\|_{\Phi, B(a, r)}+\|g\|_{\Phi, B(a, r)} .
$$


Proof. If $f=0 \mu$-a.e. or $g=0 \mu$-a.e., then we have the equality trivially; so let us assume that $f \neq 0 \mu$-a.e. and $g \neq 0 \mu$-a.e. Then, by virtue of the convexity, we have

$$
\begin{aligned}
\frac{1}{r^{n}} \int_{B(a, r)} \Phi\left(\frac{|f(x)+g(x)|}{\|f\|_{\Phi, B(a, r)}+\|g\|_{\Phi, B(a, r)}}\right) d \mu(x) \\
\leq \frac{\|f\|_{\Phi, B(a, r)}}{\|f\|_{\Phi, B(a, r)}+\|g\|_{\Phi, B(a, r)}} \\
\quad \times \frac{1}{r^{n}} \int_{B(a, r)} \Phi\left(\frac{|f(x)|}{\|f\|_{\Phi, B(a, r)}}\right) d \mu(x) \\
\quad+\frac{\|g\|_{\Phi, B(a, r)}}{\|f\|_{\Phi, B(a, r)}+\|g\|_{\Phi, B(a, r)}} \\
\quad \times \frac{1}{r^{n} \int_{B(a, r)} \Phi\left(\frac{|g(x)|}{\|g\|_{\Phi, B(a, r)}}\right) d \mu(x)} \\
\leq \frac{\|f\|_{\Phi, B(a, r)}}{\|f\|_{\Phi, B(a, r)}+\|g\|_{\Phi, B(a, r)}} \\
\quad+\frac{\|g\|_{\Phi, B(a, r)}}{\|f\|_{\Phi, B(a, r)}+\|g\|_{\Phi, B(a, r)}}=1 .
\end{aligned}
$$

From the definition of the quantity $\|f+g\|_{\Phi, B(a, r)}$, we obtain the inequality.

Lemma 22. If $\Phi:[0, \infty) \rightarrow[0, \infty)$ is a Young function, then

$$
\frac{1}{r^{n}} \int_{B(a, r)}|f(y)| d \mu(y) \leq C\|f\|_{\Phi, B(a, r)}
$$

for any ball $B(a, r)$ and $\mu$-measurable function $f$.

Proof. A normalization allows us to assume that $\|f\|_{\Phi, B(a, r)}=$ 1 ; our target will be to prove

$$
\frac{1}{r^{n}} \int_{B(a, r)}|f(y)| d \mu(y) \leq C .
$$

In view of the growth condition, we may suppose that $|f|$ assumes its value in $\{0\} \cup[1, \infty)$. Since $\Phi$ is a Young function, we have

$$
\Phi(t) \geq \Phi(1) t, \quad t \in\{0\} \cup[1, \infty) .
$$

Therefore,

$$
\begin{aligned}
& \Phi(1) \frac{1}{r^{n}} \int_{B(a, r)}|f(y)| d \mu(y) \\
& \quad \leq \frac{1}{r^{n}} \int_{B(a, r)} \Phi(|f(y)|) d \mu(y) \\
& \quad=\lim _{\varepsilon \downarrow 0} \frac{1}{r^{n}} \int_{B(a, r)} \Phi\left(\frac{|f(y)|}{\|f\|_{\Phi, B(a, r)}+\varepsilon}\right) d \mu(y) \leq 1 .
\end{aligned}
$$

So, we are done.

Now, we are ready for the proof of Theorem 17.
Proof of Theorem 17. In view of Lemma 21, $L^{\Phi, \phi}(\mu)$ is a normed space. So, we need to prove the completeness. To this end, we choose a sequence $\left\{f_{k}\right\}_{k=1}^{\infty}$ of $\mu$-measurable function such that

$$
\sum_{k=1}^{\infty}\left\|f_{k}\right\|_{L^{\Phi, \phi}(\mu)}<\infty
$$

Denote by $O$ the origin. Then, we have

$$
\sum_{k=1}^{\infty} \frac{1}{\phi(L) L^{n}}\left\|f_{k}\right\|_{L^{1}(B(O, L), \mu)} \leq C \sum_{k=1}^{\infty}\left\|f_{k}\right\|_{L^{\Phi, \phi}(B(O, L), \mu)}<\infty
$$

from Lemma 22. This implies that $\sum_{k=1}^{\infty}\left|f_{k}(x)\right|$ is finite $\mu$-a.e. on $B(O, L)$ for all $L \in \mathbb{N}$. Hence, $\sum_{k=1}^{\infty}\left|f_{k}(x)\right|$ is finite $\mu$-a.e. on $\mathbb{R}^{d}$. With this in mind, let us set $g(x)=\sum_{k=1}^{\infty} f_{k}(x)$ whenever the series is absolutely convergent; otherwise set $g(x)=0$.

We fix a ball $B(a, r)$. Then, we have

$$
\begin{aligned}
& \left\|g-f_{1}-f_{2}-\cdots-f_{k}\right\|_{\Phi, B(a, r)} \\
& =\inf \{\lambda>0 \\
& \quad: \int_{B(a, r)} \Phi\left(\frac{\left|g(x)-f_{1}(x)-f_{2}(x)-\cdots-f_{k}(x)\right|}{\lambda}\right) \\
& \left.\quad \times d \mu(x) \leq r^{n}\right\}
\end{aligned}
$$

$$
\begin{aligned}
\leq \inf \{\lambda>0 & \left\{\begin{array}{l}
\left.\lambda>{ }^{n}\right\} \\
\end{array}\right. \\
& \left.\int_{B(a, r)} \liminf _{K \rightarrow \infty} \Phi\left(\frac{1}{\lambda}\left|\sum_{j=k+1}^{K} f_{j}(x)\right|\right) d \mu(x) \leq r^{n}\right\}
\end{aligned}
$$$$
\leq \inf \{\lambda>0
$$

$$
\begin{aligned}
& \left.\quad: \liminf _{K \rightarrow \infty} \int_{B(a, r)} \Phi\left(\frac{1}{\lambda} \sum_{j=k+1}^{K}\left|f_{j}(x)\right|\right) d \mu(x) \leq r^{n}\right\} \\
& \leq \phi(r) \sum_{j=k+1}^{\infty}\left\|f_{j}\right\|_{L^{\Phi, \phi}(\mu)} .
\end{aligned}
$$

As a result, $g \in L^{\Phi, \phi}(\mu)$ and

$$
\left\|g-f_{1}-f_{2}-\cdots-f_{k}\right\|_{L^{\Phi, \phi}(\mu)} \leq \sum_{j=k+1}^{\infty}\left\|f_{j}\right\|_{L^{\Phi, \phi}(\mu)} .
$$

So $\sum_{j=1}^{\infty} f_{j}$ converges to $g$ in $L^{\Phi, \phi}(\mu)$.

The proof of Theorem 18 is similar; we use the embedding

$$
\|f\|_{w L^{1}(B(a, r), \mu)} \leq C\|f\|_{w L^{\Phi, \phi}(\mu)}
$$

which follows from Lemma 22. 
5.2. Proof of Theorem 19. We first concentrate on the maximal operator; we modify the argument to prove the boundedness of singular integral operators later.

The proof hinges upon the decomposition in Proposition 12, keeping the same notation as before. As for $f_{2}$, we have a pointwise estimate, so that a small modification works. Also, we normalize $\|f\|_{L^{\Phi, \phi}(\mu)}=1$.

Let us concentrate on $f_{1}$. Let us establish

$$
\gamma\left\|\chi_{\left\{M f_{1}>2 \gamma\right\}}\right\|_{\Phi, B(a, r)} \leq C
$$

for any $\gamma>0$, where the constant $C>0$ is independent of $\gamma$ and $f$. Write $\Lambda=\left\|\chi_{\left\{M f_{1}>2 \gamma\right\}}\right\|_{\Phi, B(a, r)}$. Then, we have

$$
\begin{aligned}
& \frac{\mu\left\{M f_{1}>2 \gamma\right\}}{r^{n}} \Phi\left(\frac{1}{\Lambda}\right) \\
& =\frac{1}{r^{n}} \int_{B(a, r)} \Phi\left(\frac{\chi_{\left\{M f_{1}>2 \gamma\right\}}(y)}{\Lambda}\right) d \mu(y)=1
\end{aligned}
$$

by virtue of the dominated convergence theorem. So, we have

$$
1 \leq C \frac{1}{r^{n} \gamma} \Phi\left(\frac{1}{\Lambda}\right)\left\|f \chi_{\{|f|>\gamma\}}\right\|_{L^{1}(B(a, 2 r), \mu)} .
$$

So

$$
r^{n} \leq C \int_{B(a, 2 r)} \Phi\left(\frac{1}{\Lambda}\right) \frac{|f(x)| \chi_{\{|f|>\gamma\}}(x)}{\gamma} d \mu(x) .
$$

Since

$$
\frac{|f(x)| \chi_{\{|f|>\gamma\}}(x)}{\gamma} \in\{0\} \cup[1, \infty),
$$

we have (by the convexity of $\Phi$ )

$$
r^{n} \leq C \int_{B(a, 2 r)} \Phi\left(\frac{|f(x)| \chi_{\{|f|>\gamma\}}(x)}{\gamma \Lambda}\right) d \mu(x) .
$$

In view of the doubling property, we are done with the maximal operator.

As for the singular integral operator, we combine the above proof and that of Theorem 15 . We mimic the argument above for $f_{1}$, while we use estimate (66) obtained in the proof of Theorem 15. We omit the further details.

5.3. Proof of Theorem 20. We start with the proof of a Hedberg type inequality. Let $R>0$. Then, as in (37), we have

$$
\begin{aligned}
\left|I_{\rho} f(x)\right| \leq & \int_{\mathbb{R}^{d}} \frac{\rho(|x-y|)}{|x-y|^{n}}|f(y)| d \mu(y) \\
\leq & \int_{B(x, R)} \frac{\rho(|x-y|)}{|x-y|^{n}}|f(y)| d \mu(y) \\
& +\int_{\mathbb{R}^{d} \backslash B(x, R)} \frac{\rho(|x-y|)}{|x-y|^{n}}|f(y)| d \mu(y) \\
\leq & C\left(M^{n} f(x) \phi(R)^{b-1}+\|f\|_{L^{\Phi, \phi}(\mu)} \phi(R)^{b}\right) .
\end{aligned}
$$

So we are led to

$$
\left|I_{\rho} f(x)\right| \leq C\left(M^{n} f(x)^{b}\|f\|_{L^{\Phi, \phi}(\mu)}^{1-b}+\|f\|_{L^{\Phi, \phi}(\mu)} \inf _{r>0} \phi(r)^{b}\right),
$$

as we did in Proposition 11.

So we have to prove

$$
\begin{aligned}
\frac{\gamma}{\psi(r)}\left\|\chi_{\left\{x \in B(a, r):\|f\|_{L^{\Phi, \phi}(\mu)} \inf _{r>0} \phi(r)^{b}>\gamma\right\}}\right\|_{\Psi, B(a, r)} \leq C\|f\|_{L^{\Phi, \phi}(\mu)}, \\
\frac{\gamma}{\psi(r)}\left\|\chi_{\left\{x \in B(a, r): M^{n} f(x)^{b}\|f\|_{L^{\Phi, \phi}(\mu)}^{1-b}>\gamma\right\}}\right\|_{\Psi, B(a, r)} \leq C\|f\|_{L^{\Phi, \phi}(\mu)} .
\end{aligned}
$$

As for the first inequality, we use the following observation:

$$
\begin{aligned}
& \frac{\gamma}{\psi(r)}\left\|\chi_{\left\{x \in B(a, r):\|f\|_{L^{\Phi, \phi}(\mu)} \inf _{r^{\prime}>0} \phi\left(r^{\prime}\right)^{b}>\gamma\right\}}\right\|_{\Psi, B(a, r)} \\
& \quad \leq \frac{1}{\psi(r)}\|f\|_{L^{\Phi, \phi}(\mu)} \inf _{r^{\prime}>0} \phi\left(r^{\prime}\right)^{b} .
\end{aligned}
$$

In view of the definition of $\psi$, we are done with the estimate. As for the second inequality, we proceed as follows:

$$
\begin{aligned}
& \frac{\gamma}{\psi(r)}\left\|\chi_{\left\{x \in B(a, r): M^{n} f(x)^{b}\|f\|_{L^{\Phi, \phi}(\mu)}^{1-b}>\gamma\right\}}\right\|_{\Psi, B(a, r)} \\
& =\frac{\gamma}{\phi(r)^{b}}\left(\left\|\chi_{\left\{x \in B(a, r): M^{n} f(x)\|f\|_{L^{\Phi, \phi}(\mu)}^{1 / b-1}>\gamma^{1 / b}\right\}}\right\|_{\Phi, B(a, r)}\right)^{b} \\
& =\left(\frac{\gamma^{1 / b}}{\phi(r)}\left\|\chi_{\left\{x \in B(a, r): M^{n} f(x)\|f\|_{L^{\Phi, \phi}(\mu)}^{1 / b-1}>\gamma^{1 / b}\right\}}\right\|_{\Phi, B(a, r)}\right)^{b} \\
& \leq C\|f\|_{L^{\Phi, \phi}(\mu)} .
\end{aligned}
$$

Here, for the last inequality, we used Theorem 19.

\section{Conflict of Interests}

The authors declare that there is no conflict of interests regarding the publication of this paper.

\section{Acknowledgments}

The authors thank Professor E. Nakai of Ibaraki University for his useful comments on the original paper. The first and second authors are supported by ITB Research and Innovation Program 2013. The third author is partially supported by Grant-in-Aid for Scientific Research (C), no. 24540174, Japan Society for the Promotion of Science. The fourth author is supported by Fundamental Research Program 2013, Directorate General of Higher Education, Ministry of Education and Culture, Indonesia. 


\section{References}

[1] F. Nazarov, S. Treil, and A. Volberg, "Cauchy integral and Calderón-Zygmund operators on nonhomogeneous spaces," International Mathematics Research Notices, vol. 1997, no. 15, pp. 703-726, 1997.

[2] F. Nazarov, S. Treil, and A. Volberg, "Weak type estimates and Cotlar inequalities for Calderón-Zygmund operators on nonhomogeneous spaces," International Mathematics Research Notices, vol. 1998, no. 9, pp. 463-487, 1998.

[3] F. Nazarov, S. Treil, and A. Volberg, "The Tb-theorem on nonhomogeneous spaces," Acta Mathematica, vol. 190, no. 2, pp. 151$239,2003$.

[4] J. Verdera, "The fall of the doubling condition in CalderónZygmund theory," in Proceedings of the 6th International Conference on Harmonic Analysis and Partial Differential Equations, pp. 275-292, Publicacions Matematiques, 2002.

[5] D. E. Edmunds, V. Kokilashvili, and A. Meskhi, Bounded and Compact Integral Operators, vol. 543 of Mathematics and Its Applications, Kluwer Academic Publishers, London, UK, 2002.

[6] G. H. Hardy and J. E. Littlewood, "Some properties of fractional integrals. I," Mathematische Zeitschrift, vol. 27, no. 1, pp. 565606, 1928.

[7] S. L. Sobolev, "On a theorem in functional analysis," Matematicheskiu Sbornik. Novaya Seriya 4 , vol. 46, pp. 471-497, 1938, English translation in American Mathematical Society Translations Series 2, vol. 34, pp. 39-68, 1963.

[8] V. Kokilashvili, "Weighted estimates for classical integral operators," in Nonlinear Analysis, Function Spaces and Applications, vol. 4 of Teubner-Texte zur Mathematik, pp. 86-103, Teubner, Leipzig, Germany, 1990.

[9] E. M. Stein, Singular Integrals and Differentiability Properties of Functions, Princeton Mathematical Series no. 30, Princeton University Press, Princeton, NJ, USA, 1970.

[10] Eridani, "On the boundedness of a generalized fractional integral on generalized Morrey spaces," Tamkang Journal of Mathematics, vol. 33, no. 4, pp. 335-340, 2002.

[11] H. Gunawan, "A note on the generalized fractional integral operators," Journal of the Indonesian Mathematical Society, vol. 9, no. 1, pp. 39-43, 2003.

[12] E. Nakai, “On generalized fractional integrals," Taiwanese Journal of Mathematics, vol. 5, no. 3, pp. 587-602, 2001.

[13] Y. Mizuta, E. Nakai, T. Ohno, and T. Shimomura, "Boundedness of fractional integral operators on Morrey spaces and Sobolev embeddings for generalized Riesz potentials," Journal of the Mathematical Society of Japan, vol. 62, no. 3, pp. 707-744, 2010.

[14] V. S. Guliyev and Y. Sawano, "Linear and sublinear operators on generalized Morrey spaces with non-doubling measures," Publicationes Mathematicae Debrecen, vol. 83, no. 3, pp. 1-17, 2013.

[15] E. Nakai, "Hardy-Littlewood maximal operator, singular integral operators and the Riesz potentials on generalized Morrey spaces," Mathematische Nachrichten, vol. 166, no. 1, pp. 95-103, 1994.

[16] D. I. Hakim and H. Gunawan, "Weak $(p, q)$ inequalities for fractional integral operators on generalized Morrey spaces of non-homogeneous type," Mathematica Aeterna, vol. 3, no. 3, pp. 161-168, 2013.

[17] J. García-Cuerva and A. E. Gatto, "Boundedness properties of fractional integral operators associated to non-doubling measures," Studia Mathematica, vol. 162, no. 3, pp. 245-261, 2004.
[18] J. García-Cuerva and J. M. Martell, “Two-weight norm inequalities for maximal operators and fractional integrals on nonhomogeneous spaces," Indiana University Mathematics Journal, vol. 50, no. 3, pp. 1241-1280, 2001.

[19] I. Sihwaningrum, S. Maryani, and H. Gunawan, "Weak type inequalities for fractional integral operators on generalized non-homogeneous Morrey spaces," Analysis Theory Applications, vol. 28, no. 1, pp. 65-72, 2012.

[20] H. L. Royden and P. M. Fitzpatrick, Real Analysis, Pearson, London, UK, 4th edition, 2010.

[21] V. S. Guliyev, "Generalized weighted Morrey spaces and higher order commutators of sublinear operators," Eurasian Mathematical Journal, vol. 3, no. 3, pp. 33-61, 2012.

[22] N. Aronszajn and K. T. Smith, “Theory of Bessel potentials. part I," Annales de l'Institut Fourier, vol. 11, pp. 385-475, 1961.

[23] S. Nagayasu and H. Wadade, "Characterization of the critical Sobolev space on the optimal singularity at the origin," Journal of Functional Analysis, vol. 258, no. 11, pp. 3725-3757, 2010.

[24] Y. Sawano and H. Wadade, "On the Gagliardo-Nirenberg type inequality in the critical Sobolev-Morrey space," The Journal of Fourier Analysis and Applications, vol. 19, no. 1, pp. 20-47, 2013.

[25] Eridani, H. Gunawan, E. Nakai, and Y. Sawano, "Characterizations for the generalized fractional integral operators on Morrey spaces," Mathematical Inequalities \& Applications.

[26] Eridani and Y. Sawano, "Fractional integral operators in generalized Morrey spaces defined on metric measure spaces," Proceedings of A. Razmadze Mathematical Institute, vol. 158, pp. 13-24, 2012.

[27] Y. Sawano and T. Shimomura, "Sobolev's inequality for Riesz potentials of functions in generalized Morrey spaces with variable exponent attaining the value 1 over non-doubling measure spaces," Journal of Inequalities and Applications, vol. 2013, article 12, pp. 1-19, 2013.

[28] Y. Sawano and T. Shimomura, "Sobolev embeddings for Riesz potentials of functions in non-doubling Morrey spaces of variable exponents," Collectanea Mathematica, vol. 64, no. 3, pp. 313-350, 2013.

[29] H. Gunawan, E. Nakai, Y. Sawano, and H. Tanaka, "Generalized stummel class and Morrey spaces," Publications de l'Institut Mathematique, vol. 92, no. 106, pp. 127-138, 2012.

[30] C. Pérez, "Two weighted inequalities for potential and fractional type maximal operators," Indiana University Mathematics Journal, vol. 43, no. 2, pp. 663-683, 1994.

[31] D. I. Hakim, Weak type inequalities for fractional integral operators on generalized non-homogeneous Morrey spaces [M.S. thesis], Institut Teknologi Bandung, Bandung, Indonesia, 2013.

[32] Y. Sawano, "Generalized Morrey spaces for non-doubling measures," Nonlinear Differential Equations and Applications, vol. 15, no. 4-5, pp. 413-425, 2008.

[33] E. Nakai, "Generalized fractional integrals on generalized Morrey spaces," Mathematische Nachrichten, 2013.

[34] Y. Sawano, S. Sugano, and H. Tanaka, "Generalized fractional integral operators and fractional maximal operators in the framework of morrey spaces," Transactions of the American Mathematical Society, vol. 363, no. 12, pp. 6481-6503, 2011.

[35] A. Akbulut, V. Guliyev, and R. Mustafayev, "On the boundedness of the maximal operator and singular integral operators in generalized Morrey spaces," Mathematica Bohemica, vol. 137, no. 1, pp. 27-43, 2012. 


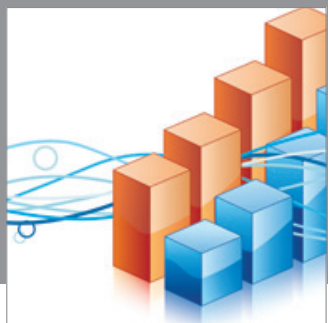

Advances in

Operations Research

mansans

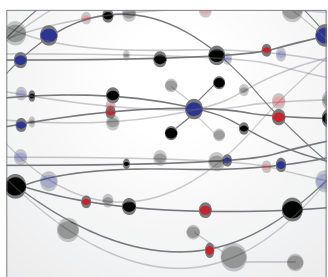

The Scientific World Journal
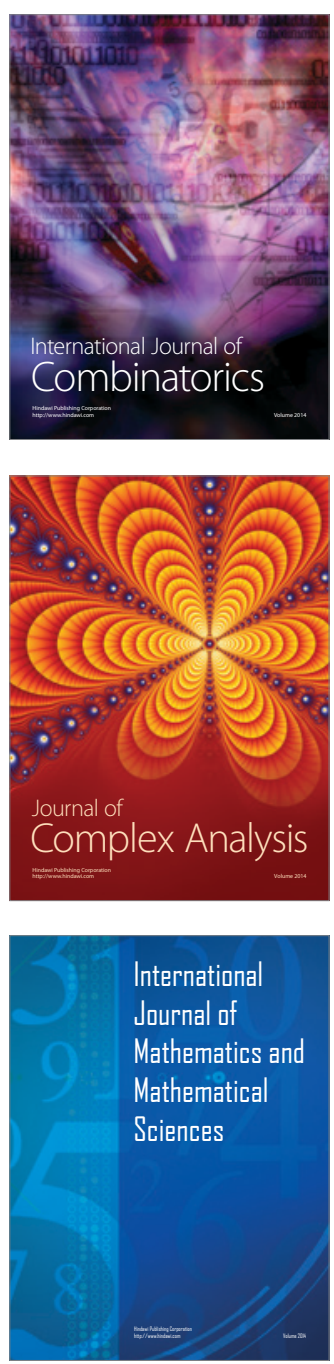
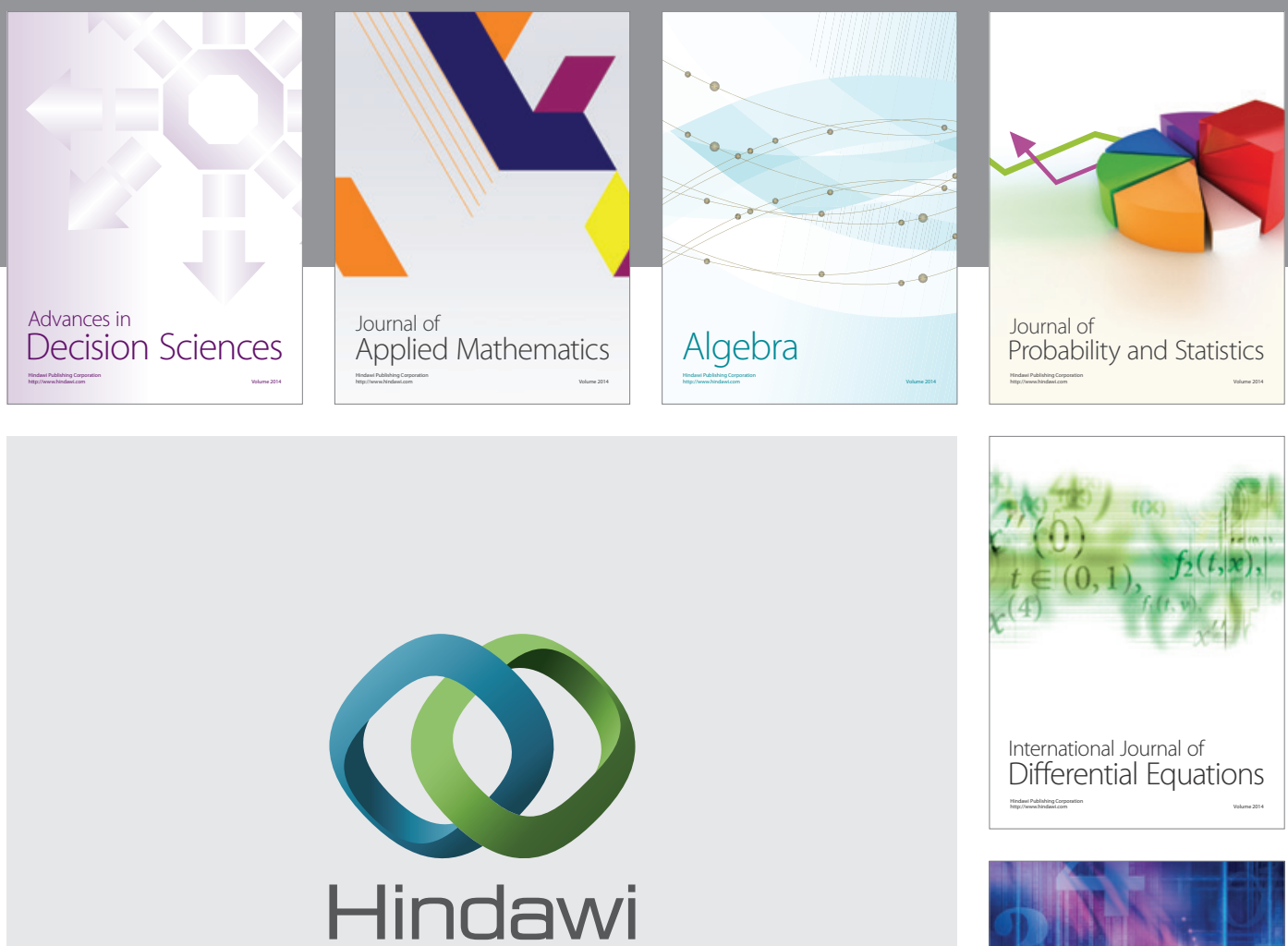

Submit your manuscripts at http://www.hindawi.com
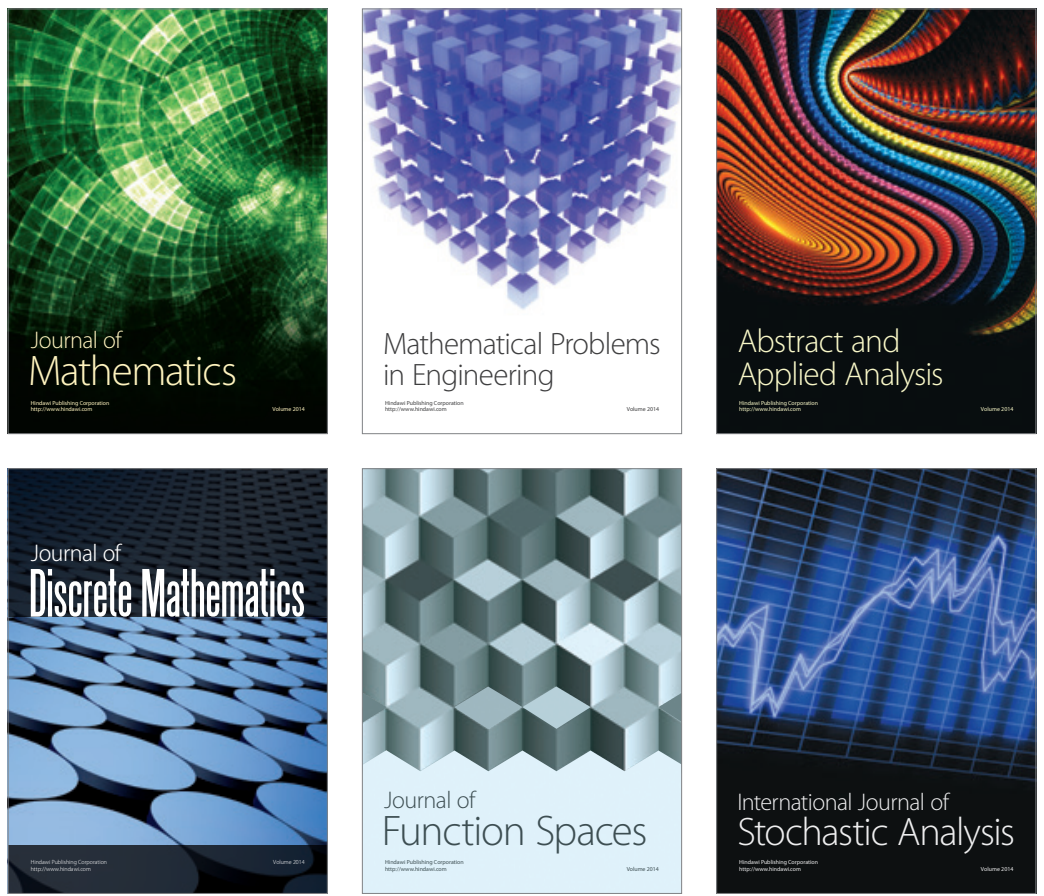

Journal of

Function Spaces

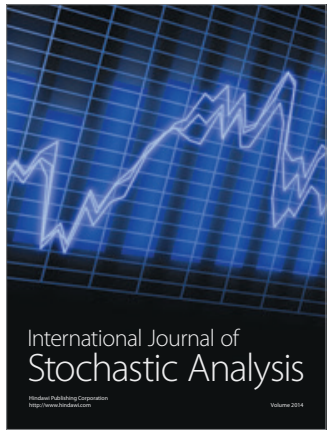

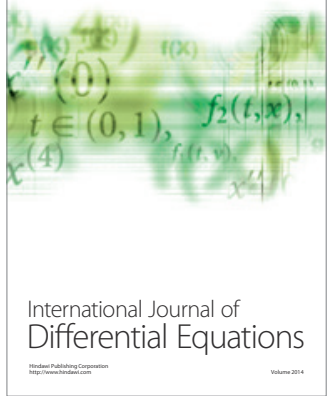
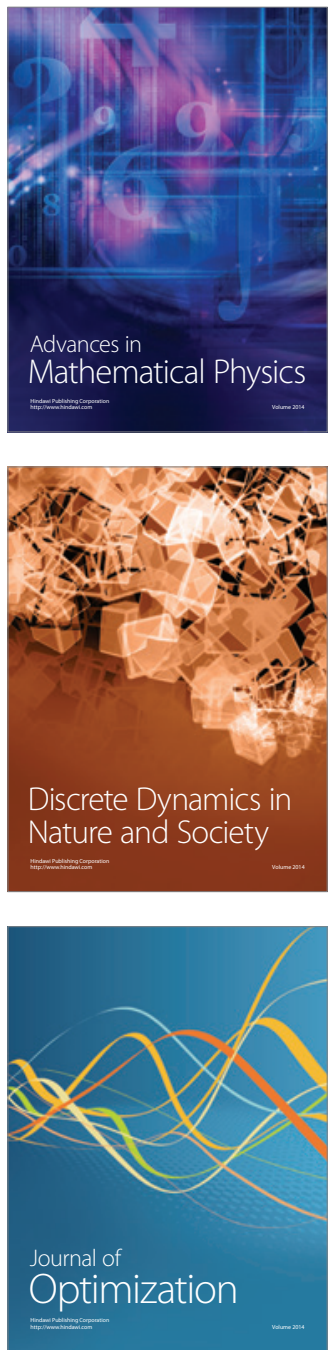